\title{
INSTITUTIONAL TRADING AND STOCK PINNING
}

Ge Zhang, William Paterson University, Wayne, NJ, U.S.A. Francis Cai, William Paterson University, Wayne, NJ, U.S.A. Lianzan Xu, William Paterson University, Wayne, NJ, U.S.A.

dx.doi.org/10.18374/JIFE-19-3.3

\begin{abstract}
This paper investigates the impact of institutional trading activity on stock pinning effect for both monthly and weekly equity options. After the introduction of weekly options over seven years, it shows the pinning effect with weekly options is stronger in comparison to monthly options. Furthermore, this paper shows that stock pinning remains pervasive on the expiration days and the effect is powerful with higher concentrated firm proprietary trading. We explore and find evidence that hedge rebalancing by firm traders might be a possible cause for stock pinning on option expiration days. The higher the fraction of firm trading, the more significant the clustering effect is.
\end{abstract}

Keywords: Stock Pinning, Weekly Options, Institutional trading, stock clustering 